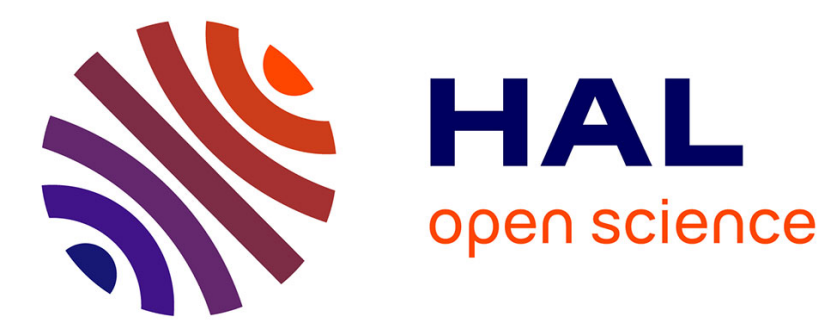

\title{
La lutte contre le feu en forêt domaniale de Fontainebleau
}

\author{
Xavier de Buyer
}

\section{To cite this version:}

Xavier de Buyer. La lutte contre le feu en forêt domaniale de Fontainebleau. Revue forestière française, 1975, 27 (S), pp.529-532. 10.4267/2042/21022 . hal-03396376

\section{HAL Id: hal-03396376 https://hal.science/hal-03396376}

Submitted on 22 Oct 2021

HAL is a multi-disciplinary open access archive for the deposit and dissemination of scientific research documents, whether they are published or not. The documents may come from teaching and research institutions in France or abroad, or from public or private research centers.
L'archive ouverte pluridisciplinaire HAL, est destinée au dépôt et à la diffusion de documents scientifiques de niveau recherche, publiés ou non, émanant des établissements d'enseignement et de recherche français ou étrangers, des laboratoires publics ou privés. 


\section{La lutte contre le feu en forêt domaniale de Fontainebleau}

Pourquoi la forêt domaniale de Fontainebleau a-t-elle son système propre de défense contre l'incendie? Pourquoi I'Office national des forêts a-t-il considérablement accru et modernisé ses moyens de lutte contre le feu dans cette forêt?

Ce sont en effet les questions que l'on peut se poser pour une forêt qui apparemment ne présente aucun des risques de ses consœurs méditerranéennes ou landaises.

Et pourtant ils existent et sont même particulièrement sérieux.

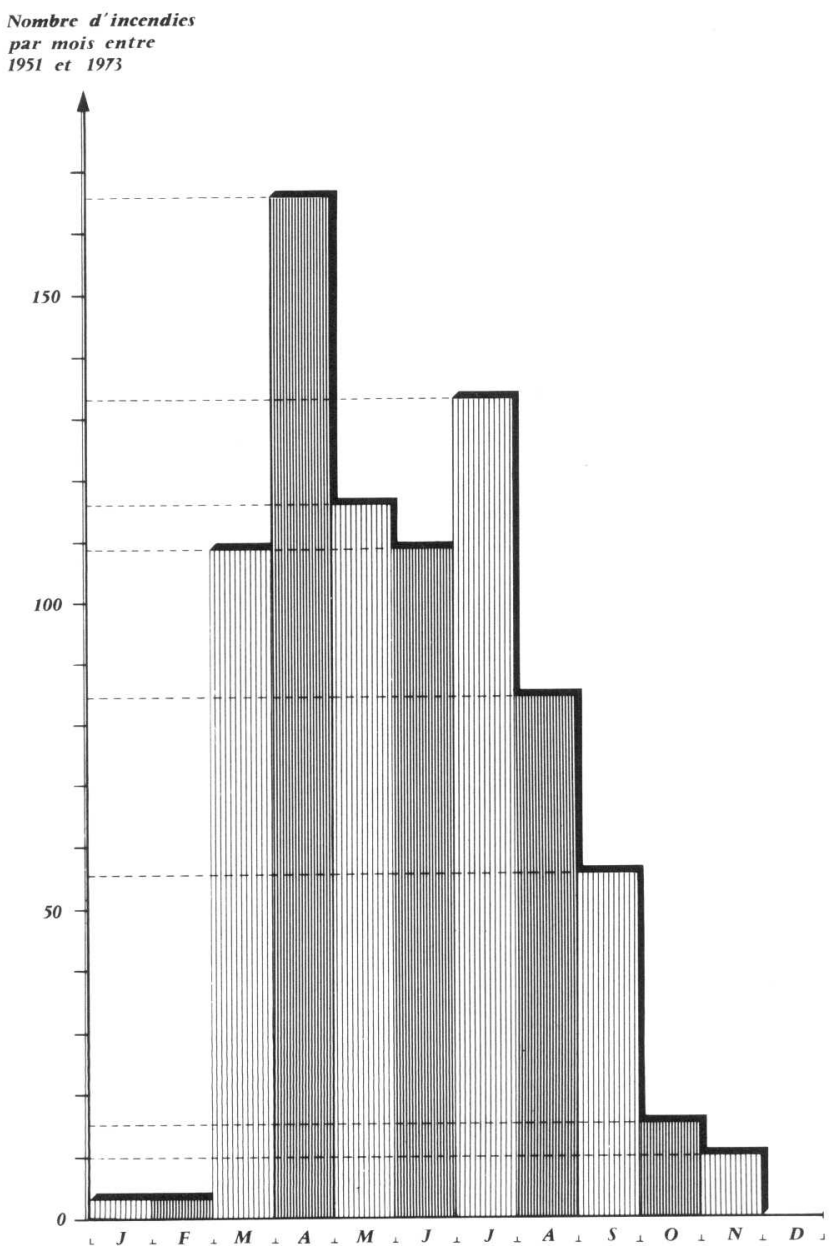

Depuis 1873 , le total des surfaces parcourues par l'incendie est de 10500 ha, ceci pour un massif boisé d'environ 20000 ha.

Ces chiffres démontrent la nécessité de protéger de façon efficace un massif dont l'immense prestige n'a d'égal que sa sensibilité à l'incendie. En outre, cette sensibilité à I'incendie due notamment aux sols sableux et secs du massif, offre la particularité d'exister tout au long de la majeure partie de l'année, ainsi qu'en témoigne le graphique $n^{\circ} 1$.
Graphique $n^{\circ} 1$

RÉPARTITION MENSUELLE DES INCENDIES 
Par ailleurs on observe que de 1875 à 1974, il y eût en moyenne 24 incendies par an. Entre 1965 et 1974 , cette moyenne passe à 33 . Elle est de 49 pour la période 1971-1974.

Cette croissance apparaît comme la simple résultante d'une fréquentation par le public de plus en plus intense, car il existe une corrélation très nette entre la densité du public et le nombre d'incendies.

Et c'est pourquoi, si le système de défense contre l'incendie de Fontainebleau a été vigoureusement renforcé, modernisé et réorganisé ces dernières années, il apparaît aussi qu'une action de prévention pour l'information du public devra être développée avec au moins autant d'énergie.

Mais nous nous bornerons dans les lignes qui suivent à décrire ce qu'est l'organisation de la défense contre l'incendie en forêt de Fontainebleau en fonction de ses particularités.

\section{CHOIX DES TECHNIQUES DE LUTTE CONTRE LE FEU}

La panoplie des techniques à mettre en œuvre en matière de défense active contre les incendies de forêts est relativement restreinte et donc les choix, assez aisés.

C'est ainsi que pour la détection, rien n'a encore remplacé l'œil humain. Ce sont donc des pylônes, comportant des guetteurs équipés de jumelles, qui assurent cette détection. Ils sont au nombre de 5 pour un massif de 20000 ha, soit 1 pour 4000 ha.

Pour les transmissions, des progrès considérables ont été réalisés avec la mise en place d'un réseau de postes émetteurs-récepteurs, infiniment plus efficaces que le téléphone. Ce dernier moyen subsiste toutefois en cas de défaillance d'un poste radio.

Ce nouveau réseau est à l'origine d'une partie des gains de temps spectaculaires de ces dernières années. En particulier, l'ensemble des postes étant immédiatement informé de l'existence d'une fumée, la recherche se fait de façon pratiquement simultanée pour l'ensemble des pylônes, et la localisation en est donc effectuée très rapidement par simple triangulation.

Les renseignements sont centralisés en un bureau de permanence situé au cœur de la forêt, en prise directe avec les moyens d'intervention propres à l'Office national des forêts et en liaison radio permanente avec les sapeurs-pompiers.

Ces moyens d'intervention sont essentiellement des camions-citernes de feux de forêts. En effet, l'absence de plan d'eau utilisable a fait éliminer les avions-citernes, tandis que l'existence d'un réseau routier important - près de $10 \mathrm{~km} / 100$ ha - se marie parfaitement à des déplacements routiers rapides. En outre, les points d'eau sont toujours à moins de quinze minutes, ce qui permet des rotations relativement rapides des camions.

Pour des raisons d'ordre esthétique, la lutte passive ne peut être organisée comme dans les Landes par exemple. En particulier, il ne peut être question d'envisager la création systématique de parefeu qui défigureraient la forêt. Seuls quelques pare-feu, étroits, ont été créés le long des routes pour protéger les reboisements artificiels récents. Par contre, la suppression du sous-étage effectuée pour des raisons sylvicoles dans les coupes en régénération est un excellent atout pour limiter la propagation du feu. Mais, toujours pour des raisons sylvicoles, il ne peut être envisagé dans les autres parcelles, d'effectuer ce travail.

II est certain que l'épandage de produits ignifugeants, effectué préventivement de façon à créer des parefeu chimiques dans les zones sensibles, est une situation a priori séduisante qui permettrait de résoudre avec élégance ce type de problèmes. Mais ces produits ne semblent pas encore opérationnels.

\section{ORGANISATION DES MOYENS DE LUTTE CONTRE LE FEU}

On le voit, les moyens de défense des forêts contre l'incendie mis en œuvre sont classiques : mais l'intérêt du système de Fontainebleau réside dans la façon dont est organisée la lutte contre le feu.

Le critère de fiabilité de cette organisation étant la diminution de la surface moyenne de chaque incendie, il est très vite apparu que les deux éléments fondamentaux qui sous-tendent cette diminution sont les suivants :

- d'une part la rapidité de l'intervention,

- d'autre part la mise en œuvre immédiate de moyens de lutte importants. 


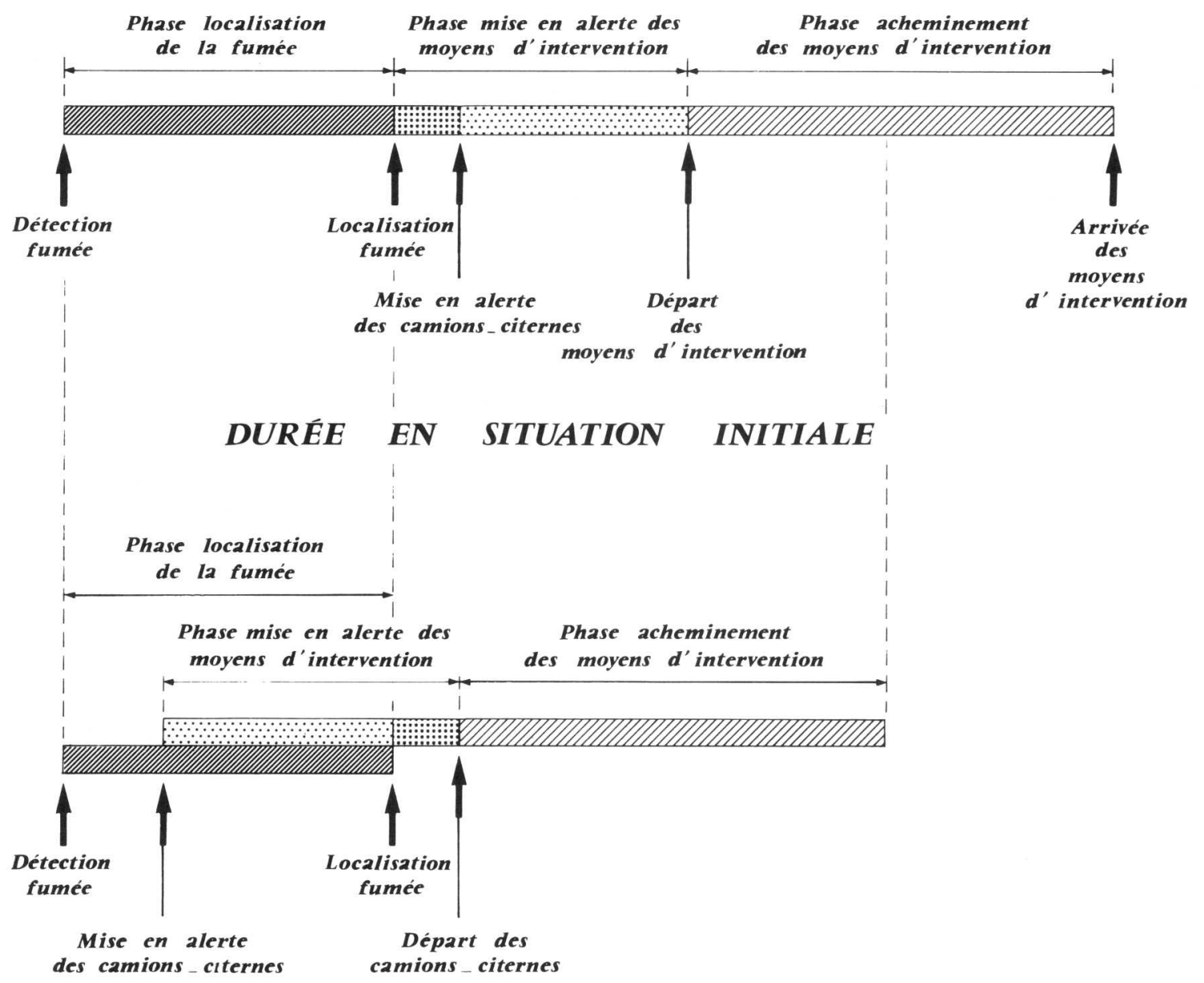

\section{DUREE RÉDUITE APRẼS SUPERPOSITION DE DEUX PHASES \\ GRAPHIQUE II}

\section{Rapidité de l'intervention}

La priorité a d'abord été donnée à la réduction des durées de toutes les phases séparant l'instant où un guetteur a découvert une fumée et l'instant où les camions-citernes sont sur les lieux. Puis, certaines phases étant susceptibles de se superposer au moins partiellement, on s'est efforcé de déclencher chacune d'entre elles le plus tôt possible dans le temps. Un exemple d'amélioration est donné dans le graphique $n^{\circ} 2$.

Voici quelques exemples concrets :

- le moteur des camions-citerne - surtout les diesels - sont tenus chauds en permanence;

- I'alerte est donnée dès qu'une fumée est repérée, ceci afin que les camions-citernes soient prêts à partir lorsque le feu aura été localisé ;

- les sapeurs-pompiers sont également immédiatement prévenus de façon à pouvoir intervenir dans l'hypothèse d'incendies susceptibles de ne pas être maîtrisés immédiatement par les moyens de l'Office national des forêts ;

- les liaisons radio propres à l'Office national des forêts s'effectuent désormais sur une fréquence particulière. Jusqu'en 1974, la fréquence utilisée étant celle des sapeurs-pompiers et leurs communica- 
tions étant fréquemment prioritaires, des pertes de temps importantes étaient constatées dans l'acheminement de nos propres messages;

- la localisation des fumées est faite par les numéros de parcelles et non plus par les lieux-dits, ceci évitant aux moyens d'intervention des erreurs de trajet. Toutes les forces d'intervention disposent désormais de la nouvelle carte I.G.N. au 1/25000e.

- I'adaptation des camions-citernes à leur mission est aussi source de gains de temps : par exemple un camion rapide sur route peut fort bien arriver après un camion plus lent mais apte à se déplacer aisément dans des conditions de terrains difficiles. D'où la gamme des camions suivants :

- un Berliet de 3000 I, très rapide sur route, relativement peu maniable sur terrains accidentés,

- trois Mercedès de $2000 \mathrm{I}$, assez rapides, légers et très maniables en tout terrain,

- un Latil de $2600 \mathrm{I}$, extrêmement puissant, à quatre roues directrices, apte à résoudre tous les cas de terrains difficiles. II s'agit d'un prototype élaboré pour les besoins propres de Fontainebleau en 1972;

- un facteur essentiel à une arrivée rapide sur les lieux est celui de la connaissance parfaite du terrain et notamment des voies de pénétration en forêt : il est bien certain que les forestiers marquent là un avantage décisif et irremplaçable.

\section{Mise en œuvre immédiate de moyens de lutte importants}

II ne sert à rien d'être rapidement sur les lieux si l'incendie n'est pas maîtrisé immédiatement. C'est pourquoi le nombre de camions-citernes a été porté de 3 à 5 en 1972. Ceci permet la mise en œuvre immédiate de trois camions-citernes de feux de forêts, les deux en réserve permettant, soit d'assurer un roulement avec les trois premiers pendant les temps de remplissage des réservoirs, soit d'aller sur un autre foyer éventuel.

En pratique on constate que l'intervention simultanée de trois camions-citernes de feux de forêts résout la majeure partie des alertes.

Toutefois malgré la proximité relative des points d'eau, on constate que les camions-citernes de feux de forêts dans la situation actuelle perdent environ $60 \%$ de leur temps en déplacements et remplissage.

C'est pourquoi I'Office national des forêts a décidé de réaliser un camion-« porteur d'eau » destiné à suivre les camions-citernes de feux de forêts. D'une contenance d'environ $10000 \mathrm{I}$, il permettra de doubler l'efficacité des camions-citernes de feux de forêts actuels. En effet non seulement les rotations pour remplissage seront pratiquement supprimées, mais encore il sera possible de ne plus laisser qu'un seul camion-citerne de feux de forêts de sécurité, disponible en cas de second incendie.

II est bien imprudent en matière d'incendie d'avancer des statistiques et d'en tirer des conclusions définitives. Bornons-nous à constater que la surface moyenne de chaque incendie était :

- de 4,3 ha pour les cent dernières années,

- de 0,9 ha pour les dix dernières années,

- de 0,3 ha pour les trois dernières années.

II semble bien que cette évolution soit parallèle à l'amélioration progressive du système de lutte contre le feu et ceci est un encouragement à en continuer le perfectionnement.

Mais il convient de noter que cette amélioration n'est pas due seulement à une évolution des matériels, mais aussi et surtout à l'action de personnels profondément motivés — forestiers et ouvriers - qui sont devenus progressivement de véritables spécialistes, professionnels de la lutte contre le feu.

En définitive, la forêt de Fontainebleau est un bon exemple de forêt périurbaine, supportant une très forte fréquentation, et comportant de ce fait des risques presque permanents d'incendie. Toutefois l'organisation qui y a été mise en place est fonction de ses caractéristiques tout à fait particulières et ne peut donc être transposée telle quelle à d'autres forêts.

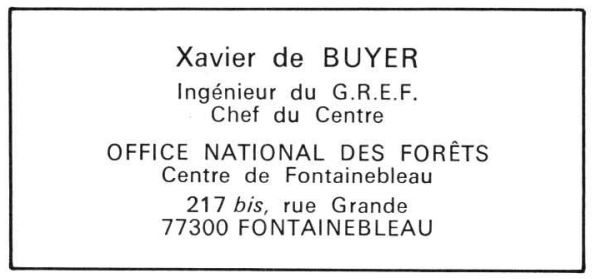

